\section{Prioridades de saúde no Brasil nos anos 1990: três políticas, muitas lições}

\author{
Cristiani Vieira Machado ${ }^{1}$
}

Palavras-chave: descentralização, política de saúde, programa saúde da família, síndrome de imunodeficiência adquirida, Sistema Único de Saúde.

\footnotetext{
Fundação Oswaldo Cruz, Escola Nacional de Saúde Pública Sérgio Arouca, Departamento de Administração e Planejamento em Saúde. Enviar correspondência para: Rua Leopoldo Bulhões 1480, sala 715, Manguinhos, CEP 21041-210, Rio de Janeiro, RJ, Brasil. Fone: +55-212598-2849; fax: +55-21-2290-0993; e-mail: cristiani@ensp.fiocruz.br
}

A reforma sanitária brasileira dos anos 1980, que teve espaço em um contexto de crise do Estado e democratização do País, representou um movimento importante no sentido da construção de um sistema de saúde universal, calcado no reconhecimento da saúde como direito de cidadania e na inserção da política de saúde em uma lógica abrangente de seguridade social. A instituição do Sistema Único de Saúde (SUS) pela Constituição de 1988 constitui um marco para o Brasil e o diferencia da maioria dos países latino-americanos, cujos sistemas de saúde, na mesma década, sofreram ajustes visando a contenção de gastos públicos, a implantação de políticas focalizadas e a expansão de mecanismos de mercado, fruto de pressões de agências internacionais e processos nacionais de Reforma do Estado de inspiração liberal.

Passada a década de 1980, marcada pela esperança de um "desenvolvimentismo democratizado" e por avanços constitucionais e político-institucionais, uma inflexão conservadora fez com que a década de 1990 no Brasil viesse a ser marcada pela convivência entre democratização e liberalização econômica (1). A agenda hegemônica de reforma do Estado nessa década foi caracterizada por: a) no plano econômico, ênfase na estabilização monetária e no controle da inflação, abertura comercial, contenção de gastos públicos, privatização de empresas estatais e fragilidade da política industrial e das estratégias de desenvolvimento de longo prazo; b) no plano administrativo, ênfase nas medidas de enxugamento da estrutura e do funcionalismo públicos, com destaque para a descentralização e novas relações entre público e privado; e c) na área social, restrições à lógica abrangente de proteção social, contenção de gastos, expansão da prestação privada (inclusive na saúde) e trajetória fragmentada das políticas sociais, com avanços institucionais esparsos e resultados limitados para a população.

Nesse contexto, o Ministério da Saúde, como autoridade sanitária nacional, construiu e buscou implementar a sua agenda de políticas de saúde, influenciado pela trajetória prévia da política da saúde, pelas diretrizes da reforma sanitária brasileira, pela agenda de reforma do Estado nacional, pelo projeto do governo federal, pelas preocupações e interesses dos dirigentes e pela interface entre os vários atores.

O objetivo deste trabalho foi identificar e analisar as políticas priorizadas pelo Ministério da Saúde brasileiro entre 1990 e 2002 e buscar compre- 
ender as possibilidades e limites dessas políticas, considerando a relação entre as agendas de reforma que repercutem sobre a saúde e a inserção da política setorial no projeto governamental. À luz da experiência brasileira, são debatidas algumas lições para a condução de políticas nacionais de saúde nos países da América Latina.

\section{MÉTODOS}

Para o mapeamento das prioridades do Ministério da Saúde, partiu-se da definição de Kingdon (2) segundo a qual "agenda" são temas que, em dado momento, mobilizam fortemente a atenção dos dirigentes governamentais e de indivíduos fora do governo ligados a esses dirigentes. A identificação das prioridades do Ministério da Saúde no período de 1990 a 2002 baseou-se nas seguintes estratégias metodológicas: a) realização de 23 entrevistas semi-estruturadas com atores envolvidos na condução da política nacional de saúde no período, abrangendo seis ministros da saúde, sete outros dirigentes de primeiro escalão do Ministério, três técnicos federais, cinco gestores de outras esferas de governo e dois representantes do Conselho Nacional de Saúde; b) análise documental; c) análise da evolução da execução orçamentária do Ministério da Saúde; e d) análise de sistemas de informações em saúde.

Para a identificação das principais prioridades do Ministério da Saúde no período estudado foram valorizados os seguintes critérios: continuidade ao longo de várias gestões ministeriais; destaque no discurso dos dirigentes entrevistados; capacidade de mobilização do ministro e de dirigentes de primeiro escalão com maior poder decisório; e repercussão significativa no financiamento da saúde.

As políticas que reuniram os atributos definidos foram analisadas quanto aos motivos para a sua entrada e continuidade na agenda principal do Ministério da Saúde e quanto às estratégias adotadas para sua condução, buscando-se elementos para compreender a direcionalidade da ação e a governabilidade da autoridade sanitária nacional.

\section{RESULTADOS}

Foram identificadas três políticas priorizadas pelo Ministério na maior parte do período de 1990 a 2002, perpassando várias gestões ministeriais: a descentralização, o Programa de Saúde da Família (PSF) e a política de combate à AIDS.

Tais políticas têm natureza e escopos distintos. A descentralização é uma diretriz de reforma do Estado; o Programa de Saúde da Família se con- figura como uma estratégia nacional de reformulação do modelo de atenção a partir da atenção básica, de indução federal e implantação descentralizada; e a política de combate à AIDS se volta para o controle de um problema de saúde específico na população e para a atenção às pessoas por ele acometidas, sob forte comando nacional no período estudado. O que fez com que tais políticas convivessem na agenda principal do Ministério da Saúde? E o que sugere a sua priorização em termos da ação federal?

Os principais motivos que favoreceram a entrada e a continuidade dessas políticas na agenda da autoridade sanitária nacional foram a confluência de diferentes agendas reformistas, a constituição de uma base social de apoio, a formação de grupos técnicos sólidos no Ministério da Saúde e o caráter atrativo como marca de governo ou de gestão ministerial.

A descentralização foi uma prioridade para o Ministério da Saúde durante todo o período estudado, favorecida pela Constituição de 1988 e pela confluência em torno do tema da agenda do movimento sanitário, que defendia a diretriz de descentralização político-administrativa de forma atrelada à democratização e à noção de sistema integrado, e da agenda liberal de reforma do Estado, para a qual a descentralização representou uma estratégia de redução do tamanho do Estado e de retração dos gastos públicos. $\mathrm{O}$ grande consenso em torno da descentralização na saúde propiciou mudanças substantivas nesse âmbito (3), embora os objetivos da descentralização e as expectativas quanto às condições para a sua efetivação tenham variado entre os grupos de atores.

O destaque dado ao PSF pelo Ministério da Saúde a partir de 1995 foi impulsionado por experiências locais prévias com modelos similares e pelo apoio de diferentes atores. A agenda da reforma sanitária brasileira já destacava a importância de mudança do modelo de atenção privatista e hospitalar hegemônico até os anos 1980. As estratégias de fortalecimento das ações básicas de saúde encontraram ainda respaldo das agências internacionais que atuam na saúde. O caráter inovador e o foco do PSF - a família e a comunidade- também fizeram dele um atraente marco de governo, pelo seu potencial de visibilidade política, contribuindo para a sua permanência na agenda das sucessivas gestões ministeriais. Outro aspecto favorável à continuidade do PSF foi a conformação de uma equipe técnica sólida no Ministério da Saúde, que permaneceu na condução do programa por cerca de 10 anos.

Já a estruturação da política nacional de combate à AIDS se deu no fim dos anos 1980, após terem sido iniciados programas estaduais em resposta ao surgimento dos primeiros casos da doença 
no país. A priorização dessa política pelo Ministério da Saúde foi favorecida pela conformação de uma base social de apoio ao enfrentamento da epidemia no âmbito nacional e internacional. No caso brasileiro, foi destacada a atuação dos movimentos sociais, particularmente as organizações nãogovernamentais ligadas à AIDS na construção da política, na pressão e na crítica às estratégias governamentais ou à sua ausência, envolvendo tanto relações de conflito como de parceria com o Estado (4). Outro aspecto fundamental para assegurar certa continuidade da política no período estudado foi a constituição de um grupo de técnicos experientes no âmbito nacional, havendo mesmo situações de permanência de coordenadores da referida política no decorrer de diferentes gestões ministeriais.

No entanto, em um contexto de conflitos entre projetos de Estado, é importante considerar as condições em que as políticas de saúde são implantadas, uma vez que isso interfere nas estratégias de condução e nos resultados. No caso brasileiro, os imperativos da redução do tamanho do Estado e da contenção de gastos públicos impostos pela agenda reformista hegemônica nos anos 1990 tiveram efeitos negativos sobre o financiamento e a gestão de recursos humanos na saúde, afetando até mesmo as políticas prioritárias para o Ministério da Saúde.

As limitações no financiamento da saúde restringem as possibilidades da descentralização de várias formas. Em primeiro lugar, aumentam o risco de cost-shifting, ou seja, de deslocamento da responsabilidade sobre os custos do sistema de saúde do governo federal para outras esferas de governo. Isso ocorre, principalmente, em momentos nos quais se observa um descompasso entre a transferência de atribuições e a transferência de recursos para as esferas subnacionais. Uma expressão disso foi $o$ apoio das autoridades econômicas dos sucessivos governos nacionais às políticas de descentralização na saúde, sem que isso se traduzisse em condições adequadas de financiamento federal para o setor. Ademais, as restrições no financiamento levaram à competição entre estados e municípios por recursos e limitaram a capacidade de superar distorções histórico-estruturais do sistema, visto que os recursos existentes são, em grande medida, comprometidos com a oferta prévia de serviços, e o aporte de novos recursos é insuficiente para a superação das desigualdades em saúde observadas entre regiões e entre grupos sociais.

O campo de recursos humanos também representou uma área de tensão entre a agenda expansiva do SUS e a agenda de reforma do Estado dos anos 1990. No período estudado, a contratação de pessoal por estados e municípios para dar conta da expansão dos serviços e ações de saúde em face da descentralização foi limitada por legislação fede- ral que restringiu os gastos com pessoal nas três esferas de governo, causando desequilíbrios. Nesse contexto, a expansão acelerada do PSF, principalmente a partir de 1998, se deu por meio do aumento de modalidades alternativas de contratação e da precariedade das relações de trabalho. Um levantamento do Ministério da Saúde mostrou que, em 2001 , somente $25 \%$ dos médicos e $29 \%$ dos enfermeiros do PSF tinham vínculos e direitos trabalhistas adequados (5). No que diz respeito à formação dos profissionais de saúde, destacam-se as incongruências entre o modelo de formação hegemônico no país e o perfil desejado para os profissionais da atenção básica, cuja superação é dificultada pela precária integração entre as políticas sociais.

As características estruturais da federação brasileira e a configuração prévia do sistema de saúde também geraram dificuldades. A ênfase na municipalização, embora coerente com as diretrizes da reforma sanitária e com a Constituição de 1988, esbarrou no perfil dos municípios brasileiros, a grande maioria de pequeno porte, de baixo dinamismo econômico e dependente das transferências federais. Nessa situação, o risco de fragmentação excessiva do sistema de saúde colocou desafios à conformação de uma rede integrada e resolutiva de serviços e à consolidação dos demais princípios do SUS.

A rápida expansão do $\mathrm{PSF}$, cuja cobertura populacional cresceu de 0,7\% em 1995 para $29 \%$ em 2002, sob forte indução do Ministério da Saúde, evidenciou algumas dessas dificuldades, na medida em que a reestruturação do modelo de atenção, pautada pela integralidade, exige recursos adequados, profissionais capacitados e um sistema organizado de referências para serviços de saúde dos diversos níveis de complexidade (inclusive entre municípios). Do contrário, corre-se o risco de confundir o PSF com uma estratégia de atendimento primário simplificado para populações carentes. Não parece ter sido esse o propósito da política conduzida pelo Ministério da Saúde no período estudado, como sugerem os achados da presente pesquisa e outros trabalhos (6). Entretanto, a expansão acelerada do PSF trouxe sérios riscos nesse sentido, face às diferentes condições estruturais dos diversos estados e municípios brasileiros, às distintas configurações locais do programa $(7,8)$ e às lacunas da política nacional de saúde.

Depreende-se dessa discussão uma característica comum à condução das três políticas priorizadas pelo Ministério da Saúde: a sua expansão em um contexto político-econômico desfavorável ocorreu sob forte indução federal e levou à adoção de estratégias especificamente direcionadas, com vistas a contornar ou a reduzir problemas que, na realidade, atingiam o conjunto do sistema de saúde. 
Foram observados, algumas vezes, esforços tardios de correção de rumos.

Assim, na segunda metade da década, os incentivos à regionalização (com vistas a evitar a fragmentação excessiva do sistema de saúde, decorrente da municipalização sob baixa coordenação intergovernamental) esbarraram nos traços estruturais da federação brasileira, na ênfase municipalista da base de apoio ao SUS e nas distorções do modelo de intervenção federal-caracterizado pela combinação entre, de um lado, segmentação e autoritarismo na condução das políticas, e de outro, insuficiente atuação em áreas estratégicas da política nacional.

A expansão do PSF envolveu inovações específicas no modelo de atenção, na organização do sistema e nas modalidades de financiamento das ações de saúde, o que, para alguns autores, permitiu identificar o programa como instrumento de reforma incremental da política de saúde brasileira (9). A prioridade do PSF na agenda do Ministério da Saúde se manifestou pela adoção de um incentivo financeiro específico, com aumento de cerca de 900\% no aporte de recursos entre 1995 e 2002 e crescimento da participação do PSF nos gastos com atenção básica de 5,2\% em 1994 para 25,2\% em 2002. Manifestou-se, também, pela implantação de pólos de capacitação em saúde da família a partir de 1997 (10), representando a iniciativa mais concreta do Ministério para formar recursos humanos em saúde no período estudado. Outras expressões da priorização dessa política foram os instrumentos gerenciais direcionados para o PSF (incluindo um sistema de informações próprio) e as estratégias diferenciadas de assistência para o PSF a partir de 2001. Tais estratégias incluíram a distribuição de medicamentos diretamente para as equipes de programa e a incorporação às equipes de profissionais de saúde bucal, como tentativas de contornar, no âmbito do programa, algumas das principais lacunas do SUS nos anos 1990.

Assim como no caso do PSF, a política brasileira de combate à AIDS, ao se pautar pelas diretrizes de universalidade e integralidade, apresentou peculiaridades significativas. Uma delas é a atuação das ONGs como prestadoras de serviços, principalmente no âmbito da prevenção. Essa atuação foi impulsionada por dois convênios com o Banco Mundial, vigentes entre 1994 e 2002, cujos recursos foram, em grande parte, direcionados para a remuneração das atividades dessas organizações. Porém, a mais emblemática peculiaridade é a firme decisão de articular a prevenção e o tratamento nos diversos níveis, incluindo a distribuição pública de todos os medicamentos anti-retrovirais necessários aos pacientes com indicação terapêutica, em dissonância com a recomendação do Banco Mundial (11) e de forma mais abrangente que em vários outros países. Tal opção expõe dilemas relacionados à inserção dos países em desenvolvimento no capitalismo mundial e coloca o Brasil no centro do debate internacional sobre as implicações da questão da propriedade intelectual para a expansão de políticas de saúde universais e abrangentes.

Nesse sentido, é interessante comentar a concomitância de três acontecimentos no ano de 1996. $\mathrm{O}$ primeiro foi o reconhecimento internacional da eficácia da combinação de drogas anti-retrovirais no tratamento de pessoas com AIDS. O segundo foi a aprovação da lei de propriedade intelectual brasileira quanto aos direitos de patente da indústria farmacêutica, que colocou o Brasil em conformidade com os termos do Acordo TRIPS da Organização Mundial do Comércio (OMC), que trata dos aspectos dos direitos de propriedade intelectual relacionados ao comércio (12). O terceiro acontecimento foi a aprovação de uma lei brasileira específica para garantir o acesso das pessoas com AIDS aos medicamentos.

Em conseqüência, entre 1995 e 2001 os gastos federais com medicamentos para doenças sexualmente transmissíveis e AIDS aumentaram mais de $24000 \%$, enquanto os gastos com outros medicamentos para programas considerados estratégicos praticamente não aumentaram. Isso fez com que o Ministério da Saúde direcionasse esforços - eventualmente bem-sucedidos- para reduzir o preço de medicamentos para a AIDS produzidos por indústrias transnacionais, tendo como trunfo a capacidade nacional de produção. Ao buscar apoio internacional para a experiência brasileira, o Ministério também contribuiu para avanços importantes, como a aprovação de uma declaração, na $4^{\circ}$ Conferência Ministerial da OMC em 2001, de que o Acordo TRIPS não deve se sobrepor aos interesses de saúde pública dos estados-membros (13). Isso pode vir a favorecer os países em desenvolvimento nas negociações com a indústria farmacêutica para a adoção de políticas de preços diferenciados, na adequação de suas legislações e na utilização do licenciamento compulsório nos casos pertinentes.

Essa linha de ação é importante, mas talvez não suficiente para livrar o país de sua dependência em relação às importações de novos medicamentos, ou de sua vulnerabilidade face aos altos preços que continuam a ser praticados pela indústria farmacêutica transnacional, comprometendo a possibilidade de acesso da população aos medicamentos necessários para a sua saúde. Decorre daí outra linha importante de ação, que é o fortalecimento da produção nacional, o que propiciou, no caso brasileiro, uma redução expressiva do preço de vários medicamentos para a AIDS não-patenteados (14) e foi importante como instrumento de pressão para a redução de preços de drogas patenteadas. 
No final do período estudado, o "modelo brasileiro" de combate à AIDS tinha apoio da sociedade brasileira e reconhecimento internacional. Dados oficiais sugerem uma redução da mortalidade e da morbidade associada à doença. Além dos benefícios em termos de sobrevida e bem-estar -que por si só justificam a política em curso- o Ministério da Saúde apresentou estimativas de redução de custos com internações e tratamento de infecções oportunistas em função do tratamento combinado com anti-retrovirais (15).

$\mathrm{O}$ avanço das três políticas priorizadas pelo Ministério da Saúde expôs lacunas da política nacional de saúde não equacionadas. Destaca-se, nesse sentido, a insuficiente articulação com outras políticas públicas na esfera econômica e social, com repercussões adversas principalmente para a provisão de insumos e a gestão de recursos humanos na saúde. Também merece atenção a inexistência de uma política de investimentos abrangente e de longo prazo, predominando os investimentos federais baixos e intermitentes, dependentes de convênios com agências internacionais ou de emendas pontuais ao orçamento oriundas do legislativo. Outro problema é a limitada consideração da diversidade das regiões do País e das variações entre municípios no desenho das políticas, levando a opções controversas, como a recente ênfase na expansão do PSF em regiões metropolitanas, cujas peculiaridades não parecem favoráveis a modelos de atenção fortemente orientados para a adscrição de clientela. Tais lacunas prejudicam a consolidação do SUS e a atuação do Ministério da Saúde na redução das desigualdades em saúde, que seria uma das principais finalidades da esfera federal.

\section{CONSIDERAÇÕES FINAIS}

Na ausência de um projeto positivo e abrangente de atuação do Ministério da Saúde, a análise de políticas federais prioritárias ajuda a compreender as complexas relações entre políticas setoriais, projetos de governo e o papel do Estado na saúde. É interessante ressaltar que o desenho de três políticas priorizadas pelo Ministério da Saúde brasileiro no período estudado não foi, a princípio, incompatível com as diretrizes do SUS. No entanto, a sua implantação em um contexto político-econômico desfavorável evidencia lacunas do sistema de saúde e sugere lições para o fortalecimento da política pública de saúde nos países latino-americanos.

Uma importante lição pode ser extraída da análise da estratégia brasileira de descentralização em saúde: ainda que esse processo não esteja em conflito com as diretrizes da reforma sanitária em um primeiro momento, as condições adversas em que ocorre, o não reconhecimento de descentra- lização e centralização como duas faces de uma mesma moeda e a falta de empenho na construção de um novo papel para a autoridade sanitária nacional são expressões da influência da agenda liberal que favorecem um modelo distorcido de intervenção federal e restringem as possibilidades de redução das desigualdades em saúde.

Já as políticas de saúde da família e de combate à AIDS são estratégias sob forte condução do Ministério da Saúde, que, ao se fundarem em objetivos abrangentes em termos de cobertura e de escopo, e exigirem expansão pública, evidenciam problemas não equacionados do sistema público. $\mathrm{O}$ avanço de tais políticas requer soluções especificamente direcionadas para lidar com as adversidades e, ao mesmo tempo, expõe as fragilidades da política federal. Nesse embate, expressam-se as tensões entre a agenda setorial e os projetos de governo, bem como os limites de governabilidade do Ministério da Saúde, visto que, em uma conjuntura desfavorável à expansão da proteção social, a autoridade sanitária tende a adotar uma postura pragmática, orientada para programas específicos e por uma perspectiva de curto prazo.

Nesse contexto, é oportuno perguntar se as conquistas alcançadas no âmbito de políticas prioritárias isoladas, como as analisadas no caso brasileiro, podem vir a configurar uma situação de dependência da trajetória (path-dependence) (16), favorável à sua continuidade, ou se podem ter os seus benefícios ampliados para o conjunto do sistema de saúde e da população. Também é preciso perguntar se o enfrentamento de problemas estruturais do sistema público de saúde não exigiria a construção de um projeto nacional abrangente e o fortalecimento da atuação do Estado em áreas estratégicas para a saúde, incluindo as políticas de ciência e tecnologia e industrial e a regulação de mercados em saúde. Tal caminho, embora árduo, parece o mais promissor para a expansão da proteção social em saúde nos países latino-americanos.

\section{SYNOPSIS}

\section{Health priorities in Brazil in the 1990s: three policies, many lessons}

This paper, which focuses on the Brazilian Ministry of Health's agenda as the national health authority between 1990 and 2002, identifies and analyzes Ministry priorities. Three main policies were identified for the period: decentralization, establishment of a family health program, and the policy to combat AIDS. In general, the initial design of these policies was consistent with the guidelines of the Brazilian public health system (SUS). However, discrepancies were found between the universalistic agenda of the Brazilian 
health reform carried out in the 1980s and the hegemonic agenda of state reform that prevailed in the country in the 1990s, which had liberal roots and did not favor the expansion of government actions and comprehensive social policies. Within this unfavorable political and economic context, the development of specific health policies prioritized by the Brazilian Ministry of Health revealed unsolved problems and gaps in the public health system and limitations in the
Ministry's ability to exercise its role as national health authority.

Key words: decentralization, health policy, family health program, acquired immunodeficiency syndrome, SUS (BR), Brazil.

\section{REFERÊNCIAS}

1. Sallum B Jr. Crise, democratização e liberalização no Brasil. Em: Sallum B Jr. Brasil e Argentina hoje: política e economia. Bauru-SP: EDUSC; 2004. Pp. 7-77.

2. Kingdon JW. Agendas, alternatives and public policies. $2^{\mathrm{a}}$ ed. Nova Iorque: Harper Collins; 1995.

3. Levcovitz E, Lima LD, Machado CV. Política de saúde nos anos 90: relações intergovernamentais e papel das normas operacionais básicas. Cienc Saude Col. 2001;6(2):269-91.

4. Parker R. Construindo os alicerces para a resposta ao HIV / AIDS no Brasil: o desenvolvimento de políticas sobre o HIV/AIDS, 1982-1996. Divulgação em Saúde para Debate. 2003;27:8-49.

5. Brasil, Ministério da Saúde, Secretaria de Políticas de Saúde, Departamento de Atenção Básica. Avaliação normativa do Programa Saúde da Família no Brasil: monitoramento da implantação e funcionamento das equipes de saúde da família, 2001-2002. Brasília: Editora MS; 2004. (Série C. Projetos, Programas e Relatórios). Disponível em: http://dtr 2004.saude.gov.br/dab/caadab / documentos/avaliacao_normativa_psf. pdf. Acessado em junho de 2006.
6. Fausto MCR. Dos programas de medicina comunitária ao Sistema Único de Saúde: uma análise histórica da atenção primária na política de saúde brasileira [tese]. Rio de Janeiro: Instituto de Medicina Social da Universidade do Estado do Rio de Janeiro; 2005.

7. Brasil, Ministério da Saúde, Fundação Oswaldo Cruz, Saúde da Família. Avaliação da implementação em dez grandes centros urbanos: síntese dos principais resultados. $2^{\mathrm{a}}$ ed. Brasília: Ministério da Saúde; 2005. (Série C. Projetos, Programas e Relatórios).

8. Brasil, Ministério da Saúde, Secretaria de Políticas de Saúde, Departamento de Atenção Básica. Indicadores de monitoramento do PSF em grandes centros urbanos. Brasília: Ministério da Saúde; 2002.

9. Viana ALA, Dal Poz MR. A reforma do sistema de saúde no Brasil e o Programa de Saúde da Família. Physis. 1998;8(2): $11-48$.

10. Gil CRR, Cerveira MAC, Torres ZF Pólos de capacitação em saúde da família: alternativas de desenvolvimento de recursos humanos para atenção básica. Em: Negri B, Faria R, Viana ALD. Recursos humanos em saúde: política, de- senvolvimento e mercado de trabalho. Campinas: UNICAMP; 2002. Pp. 103-26. 11. Mattos RA, Terto V Jr, Parker R. As estratégias do Banco Mundial e a resposta à AIDS no Brasil. Divulgação em Saúde para Debate. 2003;27:81-94.

12. Bermúdez JAZ, Oliveira MA, Chaves GCO. Acordo TRIPS da OMC e os desafios para a saúde pública. Em: Bermúdez JA, Oliveira MA, Esher AFC. Acceso a medicamentos: derecho fundamental, papel del Estado. Rio de Janeiro: ENSP; 2004. Pp. 69-89.

13. Galvão J. 1980-2001: uma cronologia da epidemia de HIV/AIDS no Brasil e no mundo. Rio de Janeiro: ABIA; 2002. (Coleção ABIA-Políticas Públicas v. 2).

14. Vitória MAA. A experiência do Brasil no fornecimento e no acesso universal às drogas anti-retrovirais. Divulgação em Saúde para Debate. 2003;27:116-21

15. Basso CR. O programa nacional de DST/AIDS no SUS. Em: Barjas-Negri AL, D'Avila V. O Sistema Único de Saúde em dez anos de desafio. São Paulo: Sobravime/CEALAG; 2002. Pp. 135-62.

16. Pierson P. Increasing returns, path dependence and the study of politics. Am Pol Sci Rev. 2000;94(2):251-67. 